\title{
APPLICATION OF A CHAOTIC OSCILLATOR IN AN AUTONOMOUS MOBILE ROBOT
}

\author{
Esteban Tlelo-Cuautle ${ }^{*}$ - Hugo C. Ramos-López ${ }^{* *}$ - Mauro \\ Sánchez-Sánchez $^{* * *}$ _ Ana D. Pano-Azucena ${ }^{* * * *}$ \\ Luis A. Sánchez-Gaspariano ${ }^{* * * *}$ - José C. Núñez-Pérez ${ }^{* * * * *}$ \\ - Jorge L. Camas-Anzueto ${ }^{* *}$
}

\begin{abstract}
Terrain exploration robots can be of great usefulness in critical navigation circumstances. However, the challenge is how to guarantee a control for covering a full terrain area. That way, the application of a chaotic oscillator to control the wheels of an autonomous mobile robot, is introduced herein. Basically, we describe the realization of a random number generator (RNG) based on a double-scroll chaotic oscillator, which is used to guide the robot to cover a full terrain area. The resolution of the terrain exploration area is determined by both the number of bits provided by the RNG and the characteristics of step motors. Finally, the experimental results highlight the covered area by painting the trajectories that the robot explores.

Keywords: chaotic oscillator, operational amplifier, random number generator, autonomous robot, step motor, microcontroller
\end{abstract}

2

\section{INTRODUCTION}

In electronics, chaotic oscillators have been designed with a wide variety of active devices [1-3]. That way and from their successful realizations, they are quite suitable for many engineering applications [4-8]. For instance, traditionally the control of robot systems has been implemented using computer program units (CPUs) [9], microcontrollers [10], etc. However, recently some researchers propose using chaotic oscillators to control autonomous mobile robots [11]. That idea makes sense because a good chaotic oscillator can provide a good entropy, which is very much needed to implement a truly random number generator (TRNG). In this manner, this article shows the application of a double-scroll chaotic oscillator to control the wheels of an autonomous mobile robot, in order to explore a full terrain area where the resolution depends on both, the characteristics of the step motors and the number of bits provided by the TRNG.

The theoretical description of the double-scroll chaotic oscillator is given in the following section. Further, the remaining sections show the realization of the TRNG using the double-scroll chaotic oscillator, which is validated by performing a monobit test, and finally the TRNG is used to generate pairs of numbers that are associated to coordinates $(\mathrm{x}, \mathrm{y})$, which are visited by the autonomous mobile robot.

\section{DOUBLE-SCROLL CHAOTIC OSCILLATOR}

A continuous time chaotic oscillator possesses at least one positive Lyapunov exponent. The larger the value of this positive Lyapunov exponent the better the chaotic behavior [12]. This is one metric that guarantees a successful application of a chaotic oscillator in realizing a TRNG. That way, among all the existing chaotic oscillators, this article is focused on the application of the double-scroll chaotic attractor that is generated by the dynamical system described by

$$
\begin{aligned}
& \dot{x}=y, \\
& \dot{y}=z, \\
& \dot{z}=-a x-b y-c z+d_{1} f(x)
\end{aligned}
$$

where $x, y$ and $z$ are the state variables; $a, b, c$ and $d_{1}$ are constant coefficients and $f(x)$ is a nonlinear function that can be approximated by a piecewise-linear (PWL) one to describe the well-known chaotic oscillator based on saturated function series [13]. The PWL function can be increased systematically, as already shown in [8], where the number of saturated segments equals the number of scrolls to be generated. In such PWL function: $k$ is the slope and a multiplication factor of the saturated plateaus plateau $=n k$, where $n=$ odd integer to generate an attractor with an even number of scrolls, and $n=$ even integer to generate even scrolls. $h$ denotes the saturated delay from the center of the slope and it is established according to $h_{i}= \pm m$, where $i=1, \ldots,[($ scrolls -2$) / 2]$

\footnotetext{
* INAOE, Department of Electronics, Tonantzintla, Puebla 72840, México, ${ }^{* *}$ Instituto Tecnológico de Tuxtla Gutiérrez, Chiapas, México, *** Universidad del Papaloapan, Campus Loma Bonita, Oaxaca, México, ${ }^{* * * *}$ Universidad Politécnica de Puebla, Puebla, México, ***** IPN-CITEDI, Tijuana, México, etleloaoep.mx
} 


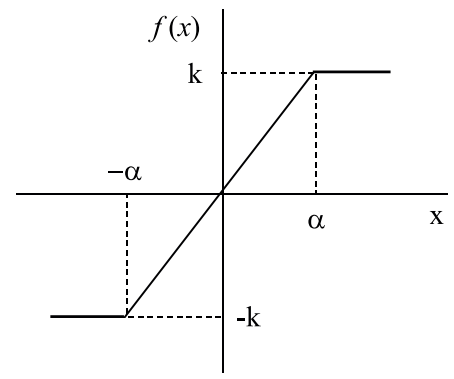

Fig. 1. 3-segments PWL function to generate 2-scrolls

and $m=2,4, \ldots,($ scrolls -2$)$ to generate an even number of scrolls; and $i=1, \ldots,[($ scrolls -1$) / 2]$ and $m=$ $1,3, \ldots,($ scrolls -2$)$ to generate odd scrolls; $p$ and $q$ are positive integers [13].

In (1), the PWL function is parameterized as shown in (2), but it needs to be scaled in order to implement the saturated function series with electronic devices [1]. That way, (3) describes the normalization of (2), providing values that can be realized with commercially available electronic devices [8].

$$
\begin{aligned}
& f(x ; k, h, p, q)= \\
& \begin{cases}(2 q+1) k, & x>q h+1, \\
k(x-i h)+2 k i, & |x-i h| \leq 1,-p \leq i \leq q, \\
(2 i+1) k, & i h+1<x<(i+1) h-1, \\
& -p \leq i \leq q-1, \\
-(2 p+1) k, & x<-p h-1 .\end{cases}
\end{aligned}
$$
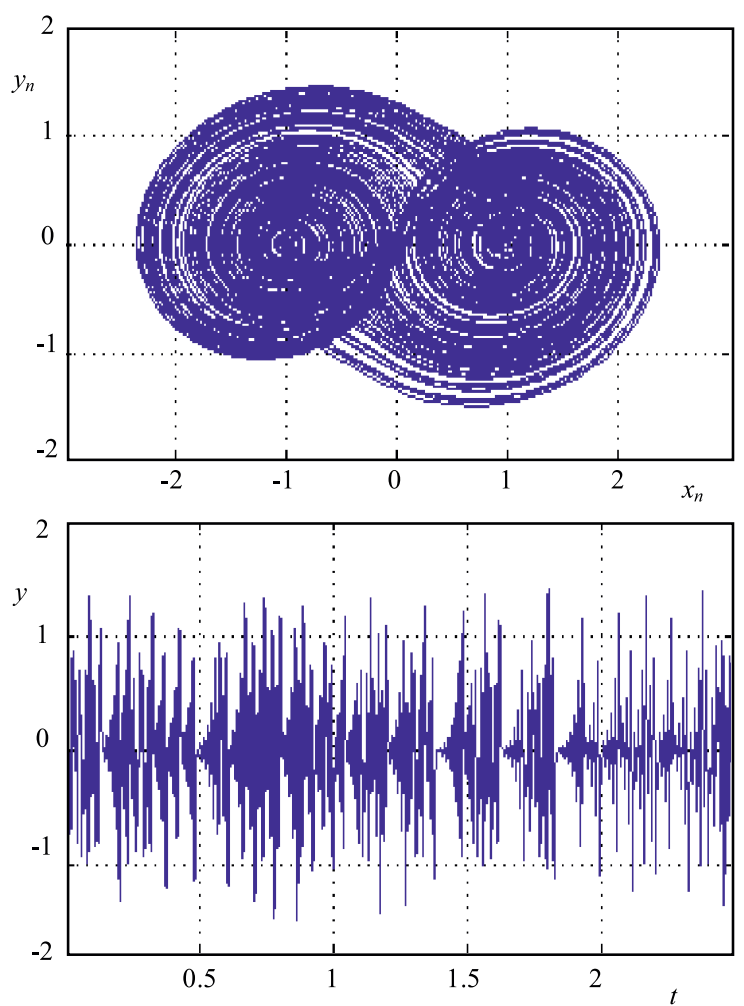

$$
\begin{aligned}
& f(x ; \alpha, k, h, p, q)= \\
& \begin{cases}(2 q+1) k, & x>q h+\alpha, \\
k / \alpha(x-i h)+2 k i, & |x-i h| \leq \alpha,-p \leq i \leq q, \\
(2 i+1) k, & i h+\alpha<x<(i+1) h-\alpha, \\
& -p \leq i \leq q-1, \\
-(2 p+1) k, & x<-p h-\alpha .\end{cases}
\end{aligned}
$$

The numerical simulation of this chaotic oscillator generating a double-scroll attractor requires the definition of a PWL function with two saturated segments, as shown in Fig. 1, where $f(x)$ in (1) is given by (4).

By setting $a=b=c=d_{1}=0.7, k=1, \alpha=$ $6.4 \times 10^{-3}, s=156.25, h=2, p=q=1$, the simulation result is given in Fig. 2 .

\section{EXPERIMENTAL VERIFICATION OF THE CHAOTIC OSCILLATOR}

The chaotic oscillator can be implemented from (1), which has the block diagram description shown in Fig. 3. Afterwards, such a diagram can be realized using operational amplifiers (opamps), as well as by using integrated circuit technology, as summarized in [1]. In this article, the chaotic oscillator is implemented as shown by Fig. 4 .

(2) The advantage of this circuit is that it can be augmented systematically to design attractors in more than one direction, as shown in [8].
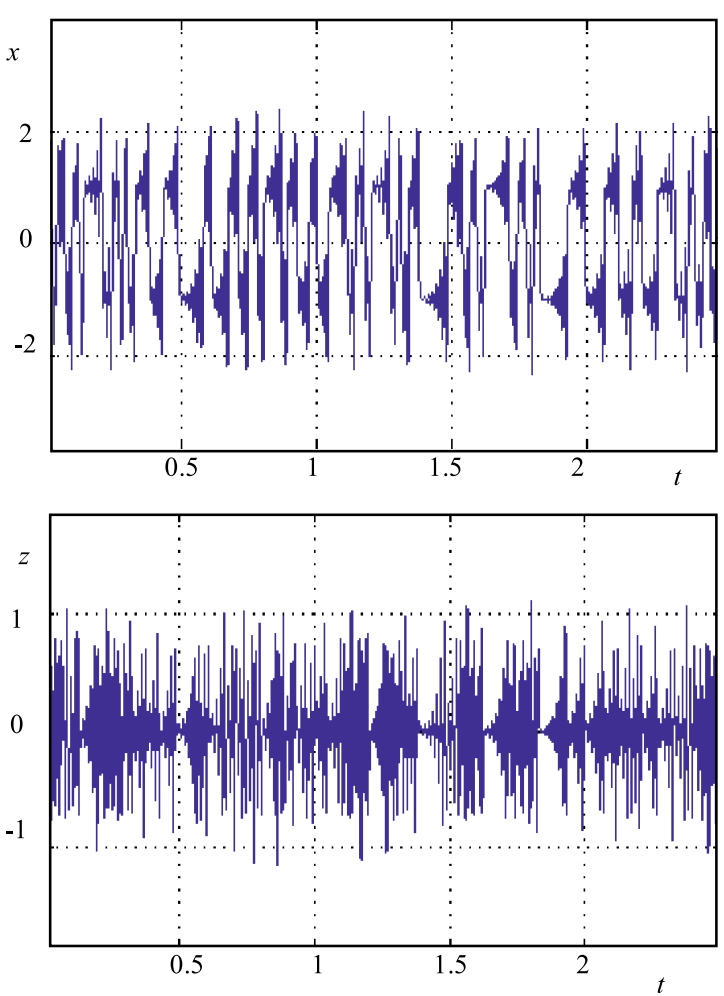

Fig. 2. Simulation results generating the double-scroll attractor, and the states $x, y$ and $z$ 


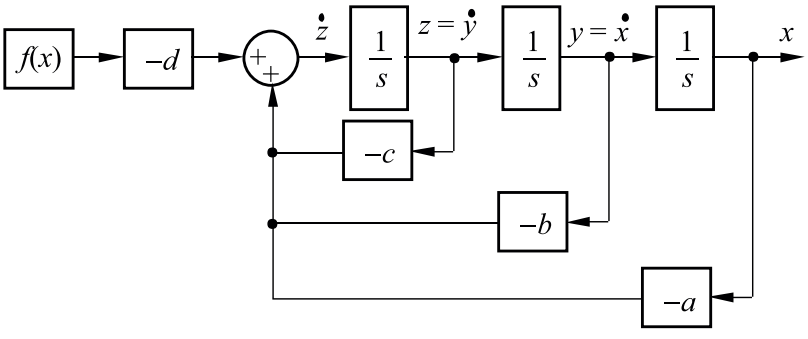

Fig. 3. Block diagram description of (1)

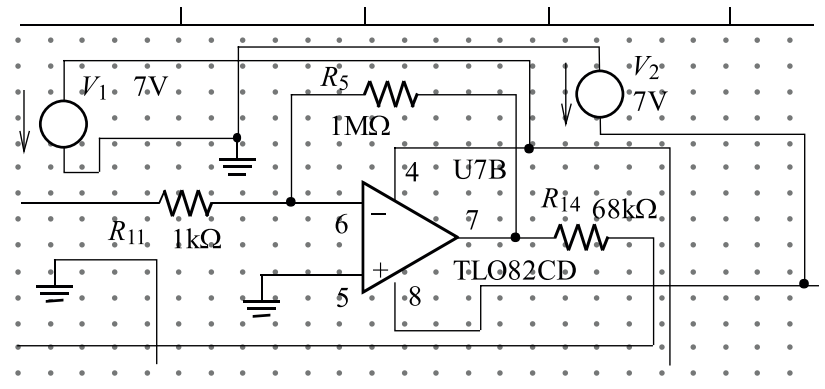

Fig. 5. Implementation of Fig. 1 with opamp

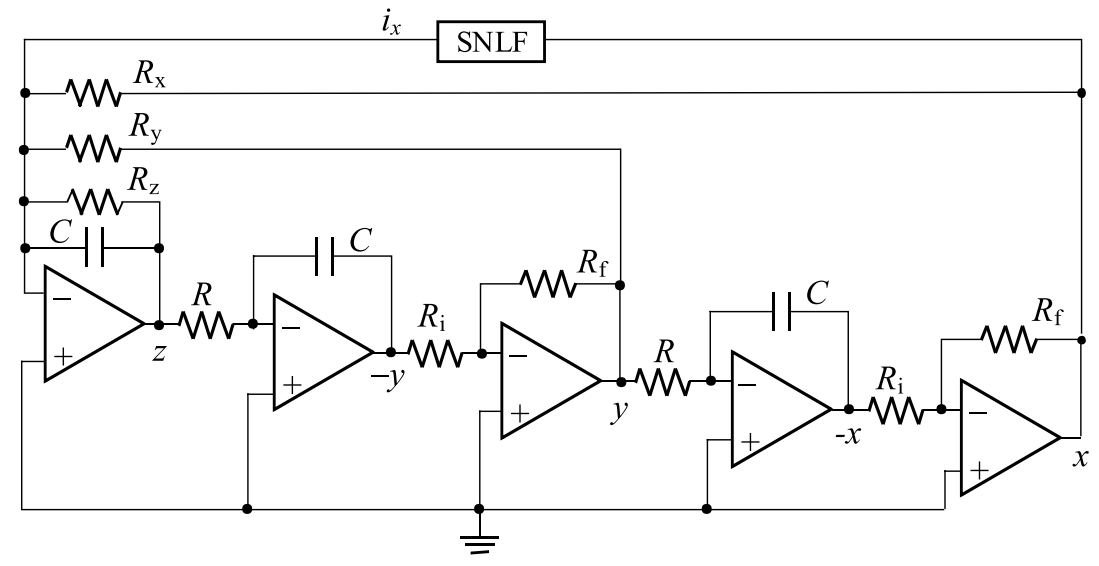

Fig. 4. Implementation of Fig. 3 using opamps

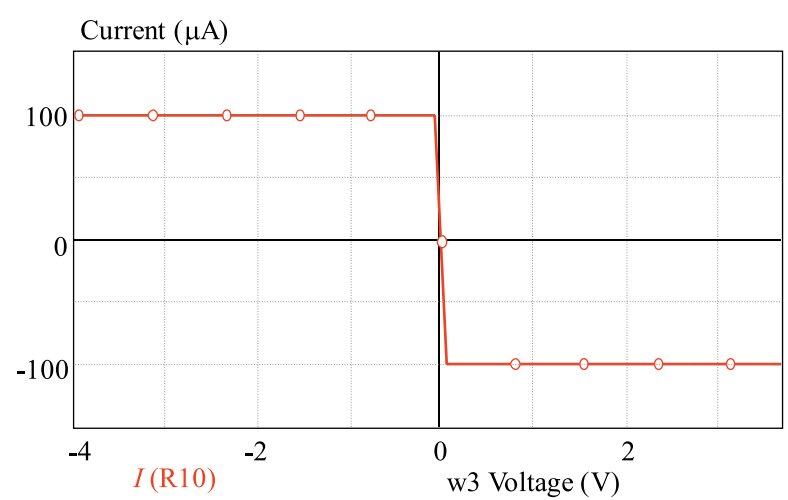

Fig. 6. DC transfer characteristic of circuit simulation from Fig. 5

$$
f(x ; \alpha, k, h, p, q)= \begin{cases}k, & x>q h+\alpha, \\ s x, & -\alpha \leq x \leq \alpha, \\ -k, & x<-p h-\alpha .\end{cases}
$$

Figure 5 shows the SPICE-like description of the PWL function using the opamp TL082, and Fig. 6 shows the circuit simulation, where the PWL function is flipped on the horizontal axis by the simulator. Finally, the experimental result is shown in Fig. 7.

\section{THE RANDOM NUMBER GENERATOR AND THE WHOLE SYSTEM REALIZATION}

Figure 8 shows the whole implementation, where: the opamp labeled by U1A is for the PWL function (as shown in Fig. 5), the opamps U1B, U2A, U2B, U3A and U3B are for the rest of the chaotic oscillator (as shown in Fig. 4), and finally, U4A, U4B and U5A are used to realize the random number generator, which basically manipulates the state variable $x$ in order to generate a sequence of bits. That way, all the nine opamps from U1A to U5A are part of the random number generator. Further, the experimental result seen at the last stage of the random number generator, ie at the output of the opamp U5A, is shown in Fig. 9.

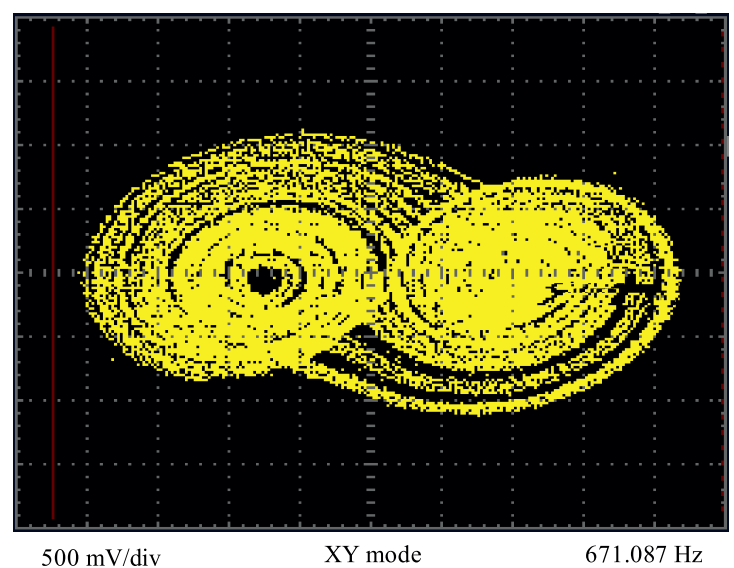

Fig. 7. Double-scroll attractor

To verify the randomness of the number generator, we used the Arduino programming language

(http://www.arduino.cc/) to capture the pulse and then to perform the monobits test with an script programmed 

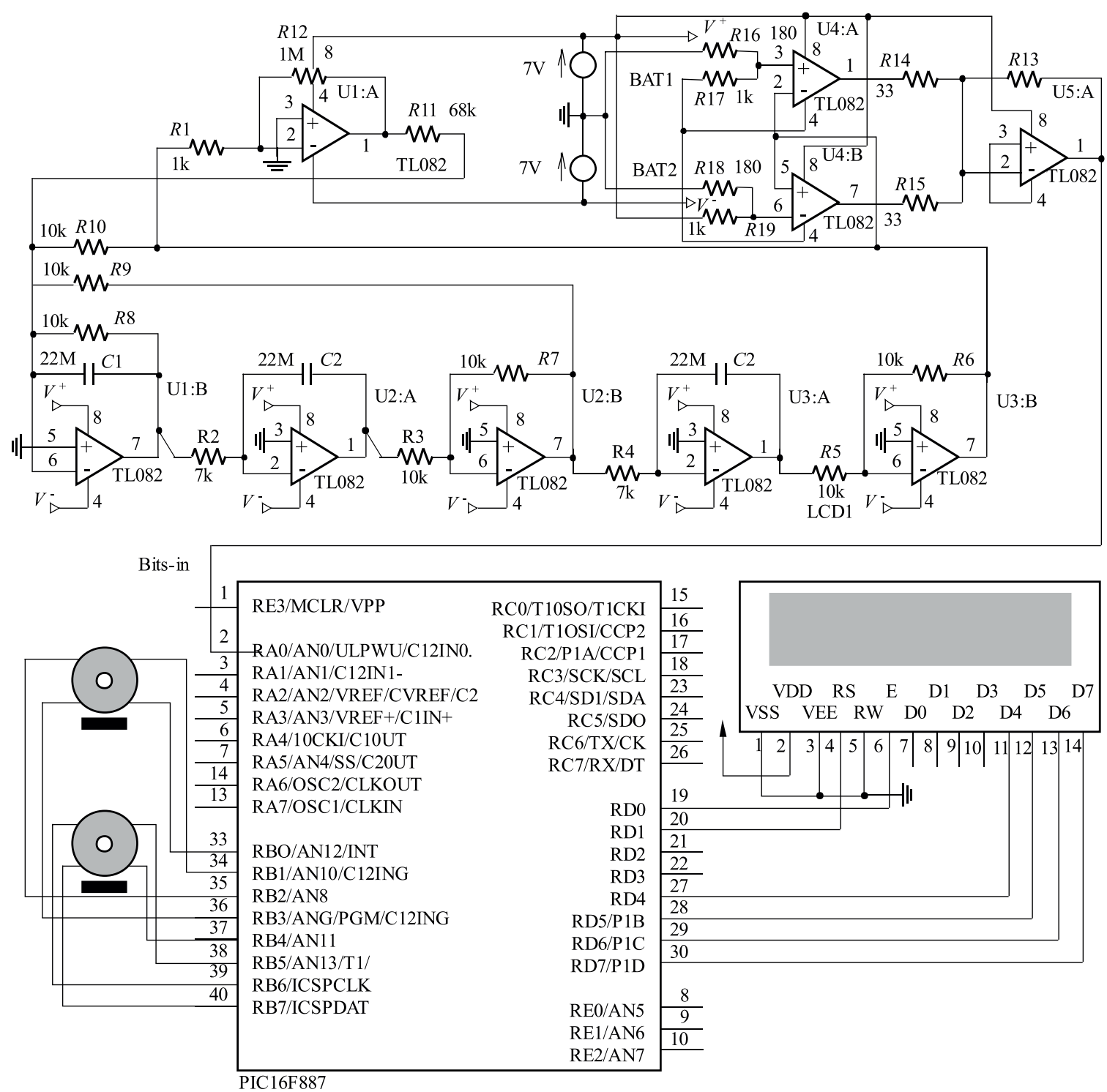

Fig. 8. Whole robot control

in MATLAB ${ }^{\mathrm{TM}}$. The monobit test consisted on recording 20000 bits to perform a comparison on the number of bits captured as 1 and 0 [11].

The monobit test requires that the range of 1's should be between 9725-10275. In our experiment, the monobit test indicated 98931 's (ones), so that our experimentally realized random number generator is suitable for controlling the wheels of the robot. That way, the pulse of the random number generator (TRNG) was captured by pin 2 of the microcontroller, as shown in Fig. 8. Afterwards, the pulses were used to control two step motors. Four bits were used for this project, so that the terrain to be explored was established as an area covering $16 \times 16$ coordinates. The proposed autonomous robot then has two wheels controlled by the TRNG, while the third wheel is just for equilibrium. The two active wheels are controlled in linear speed $v(t)$ and angular speed $w(t)$, as sketched in Fig. 10 [11]. Such movement is described by (5), where $x(t)$ and $y(t)$ are the position and $\theta(t)$ is the orientation of the robot.

$$
\left[\begin{array}{l}
\dot{x}(t) \\
\dot{y}(t) \\
\theta(t)
\end{array}\right]=\left[\begin{array}{cc}
\cos \theta t & 0 \\
\sin \theta t & 0 \\
0 & 1
\end{array}\right]\left[\begin{array}{l}
v(t) \\
\omega(t)
\end{array}\right]
$$

The kinematic control for the wheels consists of two steps: a rotation movement with angular speed around its center to point to the next position (coordinate $(x, y)$ ), according to (6), and afterwards, the robot goes to the next coordinate with constant speed, according to (7).

$$
\begin{aligned}
& {\left[\begin{array}{c}
\dot{x} \\
\dot{y} \\
\dot{\theta}
\end{array}\right]=\left[\begin{array}{c}
0 \\
0 \\
\omega
\end{array}\right],} \\
& {\left[\begin{array}{c}
\dot{x} \\
\dot{y} \\
\dot{\theta}
\end{array}\right]=\left[\begin{array}{c}
\cos \theta \\
\sin \theta \\
0
\end{array}\right][v] .}
\end{aligned}
$$

These two steps control law was programmed in MATLAB $^{\mathrm{TM}}$ using the microcontroller 16f887, as shown 


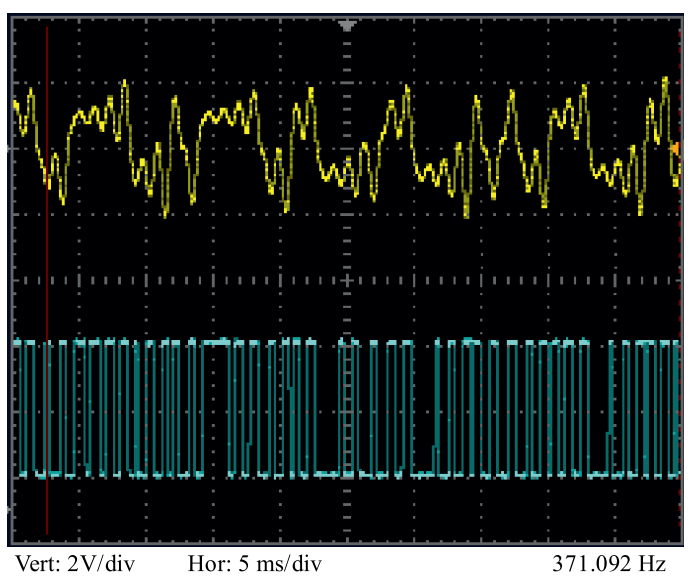

Fig. 9. Signal provided by the random number generator

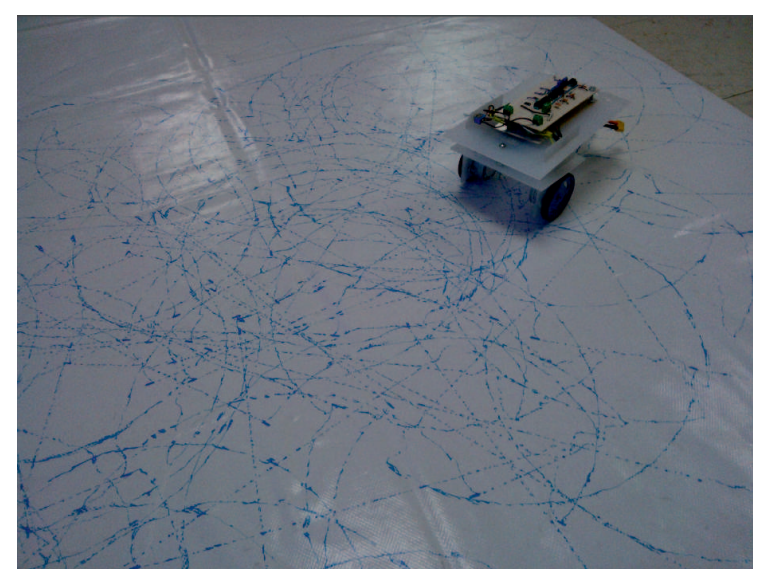

Fig. 11. Experimental result for exploring a $1.6 \mathrm{~m} \times 1.6 \mathrm{~m}$ terrain

in Fig. 8. The microcontroller captures the bits from the TRNG, which are used to set each coordinate $(x, y)$. The robot starts at coordinate $(0,0)$ and goes to the next one provided by the TRNG, according to the two steps control law.

The whole robot was realized with soft material with two step motors of 7.5 degrees, and one wheel for equilibrium, as shown in Fig. 11. The autonomous robot explored a terrain of $1.60 \mathrm{~m} \times 1.60 \mathrm{~m}$ using four bits $(16 \times 16$ coordinates $(x, y))$. The resolution using 4-bits was established to be $10 \mathrm{~cm}$. To visualize the explored terrain, a marker was tied to the tail of the robot (for correctness it must be placed between the two controlled wheels), so that one can appreciate the two steps control law. The chaotic oscillator was placed on the top and biased with $9 \mathrm{~V}$ batteries.

\section{CONCLUSIONS}

Nowadays, chaotic oscillators found many novel applications in engineering and other scientific areas. This article highlighted the application of a double-scroll chaotic oscillator for controlling an autonomous mobile robot that explores a terrain specified by the number of bits

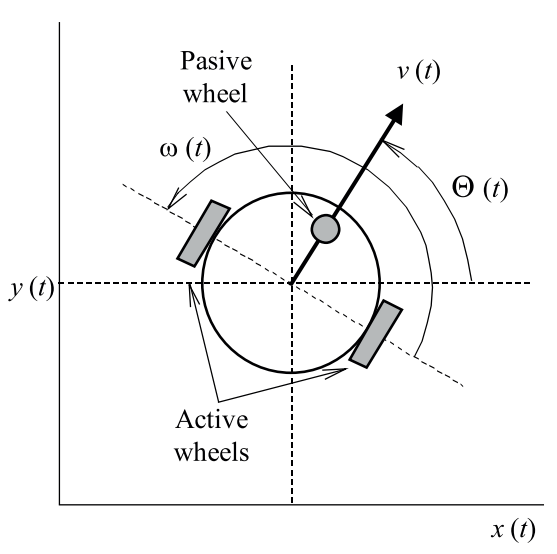

Fig. 10. Kinematic motion description

provided by a TRNG and according to the characteristics of the step motors. This application avoids the effort wasted in implementing computational hardware, ie on a CPU. In this case, the seed of the explorer robot is the chaotic oscillator that was designed with opamps, as well as its application as TRNG.

\section{Acknowledgment}

This work has been supported by CONACyT Mexico under project 131839-Y.

\section{REFERENCES}

[1] TREJO-GUERRA, R.-TLELO-CUAUTLE, E.-CARBAJAL-GOMEZ, V. H.-RODRIGUEZ-GOMEZ, G. : A Survey on the Integrated Design of Chaotic Oscillators, Applied Mathematics and Computation 219 No. 10 (2013), 5113-5122, DOI: 10.1016/j.amc.2012.11.021.

[2] GOTTHAns, T.-HRUBOŠ, Z.: Multi Grid Chaotic Attractors with Discrete Jumps, J. Electrical Engineering 64 No. 2 (2013), 118-122, DOI: 10.2478/jee-2013-0017.

[3] SANCHEZ LOPEZ, C.: A $1.7 \mathrm{MHz}$ Chua's Circuit using VMs and CF+s, Revista Mexicana de Fisica, 58 No. 1 (2012), 86-93.

[4] PÁSZTÓ, P.-PETERHUBINSKÝ, P.: Mobile Robot Navigation BASED on Circle Recognition, J. Electrical Engineering 64 No. 2 (2013), 84-91, DOI: 10.2478/jee-2013-0012.

[5] SERRANO-GUERRERO, H.-CRUZ-HERNANDEZ, C.-LOPEZ-GUTIERREZ, R. M.-CARDOZA-AVENDANO, LCHAVEZ-PEREZ, R. A.: Chaotic Synchronization in Nearest-Neighbor Coupled Networks of 3D CNNs, Journal of Applied Research and Technology 11 (2013), 26-41.

[6] CARDOZA-AVEndANO, L.-LOPEZ-GUTIERREZ, R. M.CRUZ-HERNANDEZ, C.: Encrypted Audio Transmission via Synchronized Chaotic Nd:YAG Lasers, Revista Mexicana de Fisica 58 No. 6 (2012), 472-480.

[7] NOSOV, V. R.-DOMINGUEZ, H.-ORTEGA-HERRERA, J. A.-J. A. MEDA-CAMPAÑA, J. A.: Complex Dynamics and Chaos in Commutable Pendulum, Revista Mexicana de Fisica 58 No. 1 (2012), 6-12.

[8] MUÑOZ-PACHECO, J. M.-ZAMBRANO-SERRANO, E.FELIX-BELTRAN, O.-GÓMEZ-PAVÓN, L. C.-LUIS-RAMOS, A.: Synchronization of PWL Function-Based 2D and 3D Multi-Scroll Chaotic Systems, Nonlinear Dynamics 70 No. 2 (2012), 1633-1643. 
[9] THAKUR, A.—ŠVEC, P.—GUPTA, S. K. : GPU Based Generation of State Transition Models using Simulations for Unmanned Surface Vehicle Trajectory Planning, Robotics and $\mathrm{Au}-$ tonomous Systems 60 No. 12 (2012), 1457-1471.

[10] KERNBACH, S.: Encoder-Free Odometric System for $\mathrm{Au}-$ tonomous Microrobots, Mechatronics 22 No. 6 (2012), 870-880.

[11] VOLOS, C. K.-KYPRIANDIS, I. M.-STOUBOULOS, I. N. : A Chaotic Path Planning fOr Autonomous Mobile Robots, Robotics and Autonomous Systems 60 (2012), 651-656.

[12] CARBAJAL-GÓMEZ, V. H.-TLELO-CUAUTLE, E.-FERNÁNDEZ, F. V.: Optimizing the Positive Lyapunov Exponent in Multi-Scroll Chaotic Oscillators with Differential Evolution Algorithm, Applied Mathematics and Computation 219 No. 15 (2013), 8163-8168.

[13] LU, J.-CHEN, G.-YU, X.-LEUNG, H. : Design and Analysis of Multiscroll Chaotic Attractors From Saturated Function Series, IEEE Transactions on Circuits and Systems 51 No. 12 (2004), 2476-2490

Received 15 April 2013

Esteban Tlelo-Cuautle received a BSc degree from Instituto Tecnolgico de Puebla (ITP), Mxico in 1993. He then received both $\mathrm{MSc}$ and $\mathrm{PhD}$ degrees from Instituto Nacional de Astrofsica, ptica y Electrnica (INAOE), Mxico, in 1995 and 2000, respectively. From 2001 he is Professor-researcher at INAOE. He has published 10 books, 20 book chapters, 72 journal articles and more than 120 conference papers. His research interests include systematic circuit synthesis techniques, modeling and simulation of linear and nonlinear circuits and systems, design and applications of chaotic oscillators, symbolic circuit analysis, multi-objective optimization by evolutionary algorithms, and analog/RF and mixed-signal design automation tools.

Hugo Cesar Ramos-Lopéz was born in July 9, 1990 at Pijijiapan, Chiapas, México. He got his Bachelor degree from Instituto Tecnológico de Tuxtla Gutiérrez, Chiapas, México, in 2012. His reseach interests include microcontrollers, robotics and electronic design.

Mauro Sánchez-Sánchez received the BSc degree from Instituto Tecnológico de Puebla (ITP), México in 1993. He then received both $\mathrm{MSc}$ and $\mathrm{PhD}$ degrees from Instituto Nacional de Astrofsica, Óptica y Electrónica (INAOE), México, in 2001 and 2005, respectively. He is Professor at Universidad del Papaloapan, campus Loma Bonita, Oaxaca, México. His research interests include optical fiber, communications and electronic design.

Ana Dalia Pano-Azucena received the BSc degree in Electronics and Telecommunications Engineering from Universidad Politcnica de Puebla, Puebla, Mxico, in 2013. She is currently pursuing the MSc degree with emphasis in Integrated Circuit design at Instituto Nacional de Astrofsica, Óptica y Electrónica (INAOE), México.

Luis Abraham Sánchez-Gaspariano received the $\mathrm{PhD}$ degree in electronics from the Instituto Nacional de Astrofsica, Óptica y Electrónica (INAOE), México, in 2011, on the subject of High-efficiency CMOS Power Amplifiers for RF applications. During 2009 he was a visiting scholar in the Integrated Circuit-Design Group at University of Twente, The Netherlands. Since 2011, he has been Associate Professor with the Electronics and Telecommunications department at Universidad Politécnica de Puebla, México, heading the Electronics Research Group, with a main focus on mixed-signal/RF electronics and energy harvesting/harnessing systems.

José Cruz Núñez-Pérez was born in Uruapan Michoacan, Mexico, in April 2, 1978. He received the MSc degree in electronics engineering from the National Center of Research and Technological Development (CENIDET), in Cuernavaca, Mexico, in 2003, and the PhD degree from the Institut National des Sciences Appliquées de Lyon (INSA-Lyon), Villeurbanne France, in 2007. From 2008 he is a Professor at Digital Technology Research and Development Center of National Polytechnic Institute (CITEDI-IPN), in Tijuana, Mexico. He is the Research Coordinator in Telecommunications Department at CITEDI. His research interests include digital and analog circuits design, devine physic modeling, Si/SiGe:C heterojunction bipolar transistor, VCO design, oscillator phase noise, high frequency circuits, DSP and FPGA design, circuit and system co-simulation, and electromagnetic compatibility.

Jorge Luis Camas-Anzueto received the $\mathrm{PhD}$ degree from Instituto Nacional de Astrofsica Óptica y Electrónica (INAOE), Puebla, México, in 2004. He is currently a researcher in Maestra en Ciencias en Ingeniera Mecatrnica (MCIM) of the Instituto Tecnolgico de Tuxtla Gutirrez, Chiapas, Mxico. His research interests include optical ber, ber sensors, and optoelectronics control. 